Institutional Sign In

Browse

My Settings

Get Help

Subscribe
Advertisement

Conferences > 201813 th Iberian Conference ...

\title{
Contributions to the ontology of aging, the sensitive indicators of rehabilitation nursing care, in terms of self-care, in people with respiratory disorders
}

\author{
8 Author(s) \\ Cesar Fonseca ; Joao Correia ; Manuel Lopes ; Felismina Mendes ; David... View All Authors
}

19

Full
Text Views

Text Views

\section{Export to}

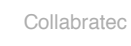

\section{Alerts}

Manage

Content Alerts

Add to Citation

Alerts

Abstract

Downl

PDF

Authors

Keywords

Metrics

More Like This

Abstract: With the increase in the average life expectancy, the appearance of chronic diseases and, in particular, in the respiratory forum and its disadvantages in the autonomy an... View more

\section{Metadata}

\section{Abstract:}

With the increase in the average life expectancy, the appearance of chronic diseases and, in particular, in the respiratory forum and its disadvantages in the autonomy and self-care of patients, brought an important paradigm for health care and, in particular, rehabilitation nursing, with implications for the development of ontologies of aging. Thus, it is imperative to systematize them in scientifically measurable indicators to demonstrate the benefit that rehabilitation nursing brings to improve patients' quality of life, thus ensuring excellence. Each area of scientific health should contribute to the construction of aging ontology. Objective: to identify indicators sensitive to nursing care of rehabilitation, in terms of self-care, in relation to those with respiratory pathology, to be integrated into the ontology of aging. Methods: A systematic review of the literature was performed using the EBSCO (full-text MEDLINE, CINAHL, Full-Text Plus, British Nursing Index), using the $\mathrm{PI}[\mathrm{C}] \mathrm{O}$ method with 6 emergent articles. Results: A total of 20 indicators were identified, including: Ability to perform activities, increase physical and functional independence, symptom management, reduction of complications, increase in quality of life, which can be allocated as a proposal for classes of the aging ontology. Conclusion: Knowledge of indicators sensitive to nursing care recognition of the importance of rehabilitation nursing in increasing self-care and autonomy for people with respiratory pathology, and research in this area is fundamental for its effectiveness and efficiency, and its integration in the ontologies of aging is fundamental.

Published in: 2018 13th Iberian Conference on Information Systems and Technologies

\section{More Like This}

Wireless system for supporting home health care of chronic disease patients 2016 IEEE Colombian Conference on Communications and Computing (COLCOM)

Published: 2016

NeuroParkinScreen - A health care system for Neurological Disorders Screening and Rehabilitation

2014 International Conference and Exposition on Electrical and Power Engineering (EPE)

Published: 2014

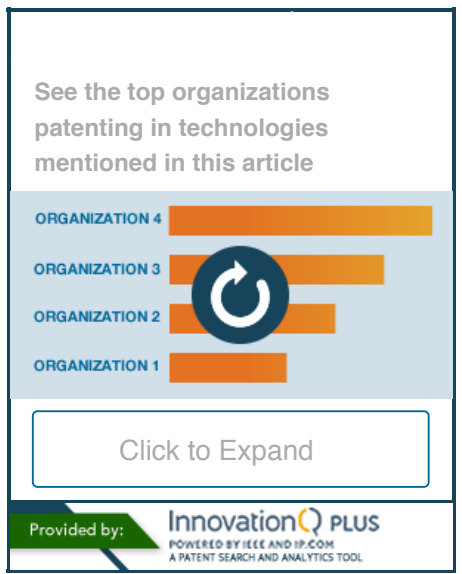

Advertisement 
Date of Conference: 13-16 June 2018

INSPEC Accession Number: 17879588

Date Added to IEEE Xplore: 28 June 2018DOI: 10.23919/CISTI.2018.8399237

ISBN Information:

Publisher: IEEE

Conference Location: Caceres, Spain

\section{IEEE Keywords}

Ontologies, Aging, Indexes, Pathology, Diseases, Object recognition

\section{INSPEC: Controlled Indexing}

diseases, health care, medical computing, medical disorders,

ontologies (artificial intelligence), patient care, patient rehabilitation, pneumodynamics

\section{INSPEC: Non-Controlled Indexing}

respiratory forum, self-care, health care, scientifically measurable indicators, scientific health, aging ontology, respiratory pathology, British Nursing Index, sensitive indicators, rehabilitation nursing care, respiratory disorders, patient quality-of-life, life expectancy, chronic diseases, EBSCO, full-text MEDLINE, CINAHL, Full-Text Plus, functional independence, physical independence, symptom management, nursing care recognition

\section{Author Keywords}

"Aging”, "Nursing interventions", "Rehabilitation nursing", "ontology", "Respiratory pathology"

Advertisement

\section{Authors}

\section{Keywords}

\section{IEEE Keywords}

Ontologies, Aging, Indexes, Pathology, Diseases, Object recognition

\section{INSPEC: Controlled Indexing}

diseases, health care, medical computing, medical disorders, ontologies (artificial intelligence), patient care, patient rehabilitation, pneumodynamics

\section{INSPEC: Non-Controlled Indexing}

respiratory forum, self-care, health care, scientifically measurable indicators, scientific health, aging ontology, respiratory pathology, British Nursing Index, sensitive indicators, rehabilitation nursing care, respiratory disorders, patient quality-of-life, life expectancy, chronic diseases, EBSCO, full-text MEDLINE, CINAHL, Full-Text Plus, functional independence, physical independence, symptom management, nursing care recognition

\section{Author Keywords}


"Aging", "Nursing interventions", "Rehabilitation nursing", "ontology",

"Respiratory pathology"

Metrics

IEEE Account

Profile Information

Purchase Details

Need Help?

Other

A not-for-profit organization, IEEE is the world's largest technical professional organization dedicated to advancing technology for the benefit of humanity.

(c) Copyright 2019 IEEE - All rights reserved. Use of this web site signifies your agreement to the terms and conditions.

US \& Canada: +18006784333

Worldwide: +17329810060

\section{IEEE Account}

»Change Username/Password

"Update Address

\section{Purchase Details \\ »Payment Options \\ »Order History}

"View Purchased Documents

\section{Profile Information}

»Communications Preferences

"Profession and Education

" Technical Interests

\section{Need Help?}

" US \& Canada: +18006784333

"Worldwide: +1 7329810060

»Contact \& Support

About IEEE Xplore | Contact Us | Help | Accessibility | Terms of Use | Nondiscrimination Policy | Sitemap | Privacy \& Opting Out of Cookies

A not-for-profit organization, IEEE is the world's largest technical professional organization dedicated to advancing technology for the benefit of humanity.

(c) Copyright 2019 IEEE - All rights reserved. Use of this web site signifies your agreement to the terms and conditions.

IEEE websites place cookies on your device to give you the best user experience. By using our websites, you agree to the placement of these cookies. To learn more, read our Privacy Policy. 